\title{
Lesões musculoesqueléticas por armas de fogo em crianças e adolescentes brasileiros*
}

\section{Firearm-Related Musculoskeletal Injuries in Brazilian Children and Teenagers}

\author{
Renato Fedatto Beraldo ${ }^{1}$ Edilson Forlin ${ }^{2}$ \\ ${ }^{1}$ Faculdade de Medicina, Universidade Federal do Paraná, Curitiba, \\ PR, Brasil \\ 2 Departamento de Ortopedia e Traumatologia, Hospital de Clínicas, \\ Universidade Federal do Paraná, Curitiba, PR, Brasil \\ Endereço para correspondência Edilson Forlin, MD, MSc, PhD, Rua \\ Buenos Aires, 1020, Curitiba, PR, 80250-070, Brasil \\ (e-mail: ediforlin@hotmail.com).
}

Rev Bras Ortop 2019;54:685-691.

\section{Resumo \\ Palavras-chave \\ - armas de fogo \\ - sistema musculoesquelético/ lesões \\ - crianças \\ - adolescente}

Objetivo Avaliar e descrever lesões musculoesqueléticas por armas de fogo em crianças e adolescentes atendidas em um pronto-socorro de grande porte.

Métodos Estudo retrospectivo avaliando os prontuários de pacientes, vítimas de armas de fogo e menores de 18 anos de idade que deram entrada ao pronto socorro do nosso hospital, de janeiro de 2014 a dezembro de 2016. Um total de 51 pacientes foi excluído por não apresentar lesões musculoesqueléticas ou outras razões, enquanto 126 foram incluídos. Os dados colhidos foram: sexo; idade; via de entrada; tipo de lesão; balas alojadas e removidas; local do corpo atingido; fraturas; complicações e sequelas; lesões associadas; tempo de internamento e de UTI; cirurgias; e óbitos.

Resultados Dos 177 pacientes menores de 18 anos que deram entrada no pronto socorro por ferimento por armas de fogo, 126 pacientes $(71,2 \%)$ atenderam aos critérios de inclusão. Foram excluídos 51 (43 por não apresentaram lesões musculoesqueléticas). Cento e sete ( $84,9 \%$ ) eram do sexo masculino e $19(15,1 \%)$ do feminino. A média de idade foi 15,5 anos (limites, 2 anos +8 meses a 17 anos +11 meses). Setenta pacientes foram internados por tempo médio de 9,6 dias, e 21 pacientes foram hospitalizados na unidade de tratamento intensivo (UTI) por 14,7 dias em média. Um total de 37 pacientes $(29,4 \%)$ foi submetido a cirurgias ortopédicas. Houve um total de 6 óbitos (4,8\%). A coxa foi a principal região atingida, em um total de 43 vezes $(24,7 \%)$. Seis pacientes tiveram lesões da medula espinhal, e oito pacientes tiveram lesões de nervos periféricos. Um total de 58 pacientes (46\%) teve 71 fraturas, e o fêmur foi o principal osso fraturado (11 pacientes; $15,5 \%)$. Um total de 52 pacientes $(41,3 \%)$ tiveram lesões associadas. Das 71 fraturas, o tratamento foi conservador em $45(63,4 \%)$ e cirúrgico em 23 (32,4\%). Três evoluíram para óbito (4,2\%).

Conclusão Os adolescentes e o sexo masculino são grupo de risco para lesões de armas de fogo, e as lesões são principalmente em membros inferiores. Menos da

Trabalho desenvolvido no Departamento de Ortopedia do Hospital do Trabalhador, Curitiba, PR, Brasil.

recebido

13 de Junho de 2018

aceito

08 de Agosto de 2018
DOI https://doi.org/

10.1055/s-0039-1697021. ISSN 0102-3616.
Copyright $\odot 2019$ by Sociedade Brasileira License terms de Ortopedia e Traumatologia. Published by Thieme Revinter Publicações Ltda, Rio de Janeiro, Brazil 
Abstract

\author{
Keywords \\ - firearms \\ - musculoskeletal \\ system/injuries \\ - child \\ - adolescent
}

metade dos pacientes apresentaram fraturas, mas muitos apresentaram lesões complexas com potencial de graves sequelas.

Objective To evaluate and describe musculoskeletal injuries by firearms in children and adolescents attended in a major trauma center.

Methods This was a retrospective study that evaluated the medical records of $<18$ years old patients who were victims of injuries by firearms and who presented to the emergency department of our hospital, from January 2014 to December 2016. A total of 51 patients were excluded for not showing musculoskeletal injures or for other reasons, while 126 were included. The collected data were: gender; age; way of admission; body site hit; fractures; complications and sequelae; associated injures; hospitalization time; surgeries; deaths.

Results Out of 126 patients included, 107 were male (84.9\%) and 19 were female (15.1\%). The mean age was 15 years and 5 months old (range: 2 anos +8 meses a 17 anos +11 meses). A total of 70 patients were hospitalized (55.6\%), with a mean hospital stay of 9.6 days, and 21 patients were hospitalized in the intensive care unit (ICU) for a mean of 14.7 days. A total of 37 patients needed orthopedic surgery (29.4\%). There were 6 deaths (4.8\%). The thigh was the most hit region, in 43 injuries (24.7\%). Six patients had spinal cord sequelae, and eight patients had peripheral nerves injuries. A total of 58 patients (46\%) had 71 fractures, and the femur was the most hit (15.5\%). A total of $52(41.3 \%)$ patients presented with associated injuries. In the 71 fractures, the treatment was conservative in 45 (63.4\%), surgical in 23 (32.4\%). Three injuries resulted in death (4.2\%).

Conclusion Adolescents and males are at-risk groups for firearms injuries, and the lesions are mainly on the lower limbs. Less than half of the patients had fractures, but many had complex lesions with potential for severe sequelae.

\section{Introdução}

A violência urbana configura-se como um dos principais problemas sociais da atualidade, e as crianças e os adolescentes são identificados como grupos etários de maior vulnerabilidade. Alguns estudos mostram a gravidade do quadro: no VIVA Inquérito de 2014, o percentual de crianças e adolescentes vítimas de violência representou 29,5\%. ${ }^{1}$ Em um estudo em Campinas, estado de São Paulo, a principal causa na cidade de morte traumática em menores de idade foi por ferimentos por armas de fogo (FAF) (47\%). ${ }^{2}$

A Pesquisa Nacional de Saúde do Escolar (PeNSE), realizada em 2009 pelo Instituto Brasileiro de Geografia e Estatística (IBGE) teve como objetivo descrever as prevalências de percepção dos escolares do $9^{\circ}$ ano do ensino fundamental sobre critérios de segurança e violência. No estudo, Curitiba foi a segunda capital que mais teve relatos de brigas envolvendo armas de fogo por estudantes do $9^{\circ}$ ano do ensino fundamental no Brasil, com 5,9\% dos relatos, ficando atrás apenas de Boa Vista, RR, com $6,4 \%$. A média das 26 capitais e do distrito federal foi de $4 \%{ }^{3}$ Segundo o Departamento de Informática do Sistema Único de Saúde do Brasil (DATASUS), entre os anos 2014 e 2016, de 220 mil óbitos por todas as causas, houve $>27$ mil óbitos por FAF no Brasil em uma faixa de até 19 anos de idade. Em Curitiba, no mesmo período e faixa etária, foram 299 (de um total de 2.226 óbitos). ${ }^{4}$
Um maior conhecimento das lesões provocadas por armas de fogo nos parece fundamental para elaborar políticas de enfrentamento à violência e estruturar um atendimento adequado às vitimas. No Brasil, há alguns estudos sobre FAF (incluindo musculoesqueléticos), ${ }^{5-9}$ mas sem o enfoque nos menores de idade, embora os números de homicídios sejam crescentes e alarmantes nessa faixa etária. ${ }^{10}$ Mesmo na literatura norte-americana, há poucos estudos, por razões relacionadas à legislação. ${ }^{11}$

O objetivo do presente trabalho é avaliar e descrever as lesões musculoesqueléticas de pacientes menores de 18 anos de idade atendidos entre 2014 a 2016 em um pronto-socorro (PS) de grande porte e em um grande centro urbano, com diagnóstico de FAF.

\section{Material e Métodos}

Estudo retrospectivo realizado em nosso hospital, que atende pacientes pelo Sistema Único de Saúde (SUS). Foram levantados prontuários e boletins de emergência pelo banco de estatísticas do Hospital do Trabalhador (HT) (nosso hospital), de pacientes menores de 18 anos de idade que deram entrada ao PS após FAF, entre janeiro de 2014 e dezembro de 2016. Foram acessados boletins de emergência (BEs) físicos e digitalizados, prontuários e radiologias físicos e digitais. $O$ estudo foi aprovado pelo Comitê de Ética e Pesquisa sob o número CAAE 58662816.6.0000.5225 e parecer número 1.800.239. 
Foram levantados todos os prontuários de pacientes que deram entrada com diagnóstico de FAF. Os critérios de inclusão foram: pacientes com ao menos uma região musculoesquelética atingida por armas de fogo, que buscavam o PS do HT como primeira procura ou advindos de outras unidades de saúde no mesmo dia do ocorrido, e que receberam alta ou faleceram nas dependências do PS. Foram excluídos 51 pacientes, dos quais 43 não apresentaram lesões musculoesqueléticas; outros 8 pacientes excluídos foram registrados como transferência de serviço, evasões, e ferimento por arma de chumbo ou por projéteis de borracha. Restaram 126 pacientes para estudo.

Os dados obtidos foram registrados no Microsoft Office Excel (Microsoft Corporation, Redmond, WA, EUA). Os acessos aos BEs, prontuários e radiologias foram realizados através do nome do paciente ou pelo número do $\mathrm{BE}$ ou do prontuário.

Os dados colhidos foram: sexo; data de nascimento; idade; entrada no PS; ferimento musculoesquelético (sim ou não); via de entrada (procura direta, ambulância ou via aérea); tipo de lesão; queixa principal; localização musculoesquelética do tiro; bala alojada; bala removida; fraturas; lesões associadas; internamento (sim ou não); data e hora de entrada e saída de internamento; data e hora de entrada e saída de UTI; tratamento ortopédico; cirurgia ortopédica; outros procedimentos; complicações; sequelas; óbito (sim ou não); evasão (sim ou não); observações (quando pertinentes para facilitar o estudo).

As lesões foram classificadas por tipo de lesão em: politrauma (PT), fratura $(\mathrm{F})$, superficial $(\mathrm{S})$, transfixante (T) e partes moles (PM). Estabelecemos algumas definições de termos. Politraumas: pacientes com lesão em $>1$ região corporal musculoesquelética. Fraturas: somente um segmento ósseo atingido (pela nossa divisão). Superficiais: lesões individuais sem perfuração. Transfixantes: apresentavam entrada e saída sem fraturas. Lesões de partes moles: lesões com perfuração, não transfixantes, e sem fraturas.

A localização musculoesquelética foi dividida em: coluna vertebral; ombro; braço; cotovelo; antebraço; punho e mão; bacia (pelve e sacro); coxa; joelho; perna; tornozelo e pé; glúteo; axila. Para serem incluídos na pesquisa, os pacientes deveriam apresentar pelo menos um FAF em uma dessas regiões. Foram excluídos três compartimentos por imprecisão dos registros.

As fraturas foram divididas por seguimentos ósseos: coluna cervical; coluna torácica; coluna lombar; ossos da mão; pelve; articulação coxo-femoral; rádio; clavícula; fêmur; tíbia (incluindo tíbia e fíbula); escápula; úmero; articulação gleno-umeral; ulna; ossos do pé; sacro.

As lesões associadas e outros procedimentos foram classificados em abdominais (incluindo vísceras e geniturinárias); torácicas (pulmão, coração e costelas); vasculares; encefálicas e faciais.

Os tratamentos de fraturas foram classificados em: "conservadores", "cirúrgicos" e "sem tratamento devido a óbito". Os tratamentos cirúrgicos existentes na amostra foram classificados em: artrodese de coluna, laminectomia descompressiva, fixação interna, fixação externa, fixações externas seguidas de fixação interna, e um tratamento concomitante de fixação externa e interna.

\section{Resultados}

Dos 177 pacientes menores de 18 anos que deram entrada no PS por FAF, 126 pacientes (71,2\%) atenderam aos critérios de inclusão. Um total de 54 foi atendido em 2014, 31 em 2015, e 41 em 2016. Quanto à sazonalidade, dividindo por semestres, a procura foi mais frequente no primeiro semestre do ano (janeiro-junho) com 76 casos $(60,3 \%)$, e $50(39,7 \%)$ no $2^{\circ}$ semestre (julho-dezembro). Um total de 107 pacientes era do sexo masculino (85\%), e 19 do feminino (15\%), com uma proporção de 5,6:1. A idade variou entre 2 anos e 8 meses a 17 anos e 11 meses, com média de 15 anos e 5 meses. A divisão de casos por idade está representada na - Tabela 1.

Um total de 106 pacientes era de Curitiba, e 20 de outras cidades da região metropolitana. Em relação à via de entrada, 86 pacientes $(68,2 \%)$ foram encaminhados ao HT por ambulância; $38(30,2 \%)$ por procura direta, e 2 pacientes $(1,6 \%)$ por via aérea. 0 tipo de lesão dos 126 pacientes foi distribuído da seguinte maneira: 37 PTs; 35 Fs; 28 PMs; 17 Ts; e 9 Ss. Dos 37 PTs, 23 envolviam Fs (62,1\%).

Um total de 122 pacientes tiveram registro e identificação se a bala foi alojada ou não. Destes, 61 (50\%) tiveram ao menos uma bala alojada em compartimentos musculoesqueléticos, sendo que 7 tiveram 2 balas alojadas, e 2 tiveram 3: um total de 72 projéteis alojados. Desses, 67 tinham registros a respeito do destino das balas, sendo que $25(37,3 \%)$ foram removidas e $42(62,7 \%)$ não foram removidas.

Tabela 1 Divisão dos pacientes por idade

\begin{tabular}{|c|c|c|}
\hline Idade (anos) & Pacientes & $\%$ \\
\hline 17 & 46 & $36,50 \%$ \\
\hline 16 & 31 & $24,60 \%$ \\
\hline 15 & 16 & $12,70 \%$ \\
\hline 14 & 14 & $11,10 \%$ \\
\hline 13 & 5 & $3,40 \%$ \\
\hline 12 & 0 & $0,00 \%$ \\
\hline 11 & 2 & $1,60 \%$ \\
\hline 10 & 3 & $2,30 \%$ \\
\hline 9 & 1 & $0,80 \%$ \\
\hline 8 & 1 & $0,80 \%$ \\
\hline 7 & 1 & $0,80 \%$ \\
\hline 6 & 2 & $1,60 \%$ \\
\hline 5 & 1 & $0,80 \%$ \\
\hline 4 & 0 & $0,00 \%$ \\
\hline 3 & 0 & $0,00 \%$ \\
\hline 2 & 2 & $1,60 \%$ \\
\hline 1 & 0 & $0,00 \%$ \\
\hline 0 & 0 & $0,00 \%$ \\
\hline
\end{tabular}


Tabela 2 Compartimentos musculoesqueléticos acometidos

\begin{tabular}{|l|l|l|}
\hline Compartimento & Lesões & $\%$ \\
\hline Coxa & 43 & $24,70 \%$ \\
\hline Perna & 22 & $12,60 \%$ \\
\hline Braço & 16 & $9,20 \%$ \\
\hline Coluna vertebral & 15 & $8,60 \%$ \\
\hline Antebraço & 15 & $8,60 \%$ \\
\hline Ombro & 14 & $8,10 \%$ \\
\hline Punho + mão & 14 & $8,10 \%$ \\
\hline Tornozelo + pé & 11 & $6,30 \%$ \\
\hline Glúteo & 11 & $6,30 \%$ \\
\hline Joelho & 9 & $5,20 \%$ \\
\hline Cotovelo & 2 & $1,10 \%$ \\
\hline Bacia (pelve + sacro) & 1 & $0,60 \%$ \\
\hline Axila & 1 & $0,60 \%$ \\
\hline
\end{tabular}

As 174 localizações musculoesqueléticas acometidas estão representadas na $\boldsymbol{- T a b e l a ~} 2$.

Um total de 58 pacientes (46\%) foi diagnosticado com fraturas. Destes, $9(15,5 \%)$ tiveram $>1$ segmento ósseo fraturado, sendo que 5 tiveram 2 segmentos, e 4 tiveram 3 segmentos fraturados. Houve um total de 71 fraturas diagnosticadas (-Tabela 3). Dessas, 45 (63,4\%) receberam tratamento conservador, $23(32,4 \%)$ receberam tratamento cirúrgico, e três $(4,2 \%)$ não foram tratadas devido a óbito.

Um total de $37(29,4 \%)$ pacientes foi submetido a cirurgias ortopédicas: $19(15,1 \%)$ para tratamento de fraturas (envol-

Tabela 3 Segmentos ósseos fraturados

\begin{tabular}{|l|l|l|}
\hline SEGMENTO ÓSSEO & FRATURAS & $\%$ \\
\hline Fêmur & 11 & $15,5 \%$ \\
\hline Tíbia $^{*}$ & 10 & $14,1 \%$ \\
\hline Coluna torácica & 8 & $11,2 \%$ \\
\hline Coluna lombar & 7 & $9,8 \%$ \\
\hline Ossos da mão & 6 & $8,4 \%$ \\
\hline Rádio & 6 & $8,4 \%$ \\
\hline Coluna cervical & 5 & $7,0 \%$ \\
\hline Úmero & 5 & $7,4 \%$ \\
\hline Ulna & 3 & $4,2 \%$ \\
\hline Pelve & 2 & $2,8 \%$ \\
\hline Articulação coxo-femora ${ }^{* *}$ & 2 & $2,8 \%$ \\
\hline Escápula & 2 & $2,8 \%$ \\
\hline Clavícula & 1 & $1,4 \%$ \\
\hline Articulação Gleno-Umeral & 1 & $1,4 \%$ \\
\hline Ossos do pé & 1 & $1,4 \%$ \\
\hline Sacro & 1 & $1,4 \%$ \\
\hline
\end{tabular}

${ }^{*}$ Quatro concomitantemente na fibula.

${ }^{*}$ Os dois no acetábulo. vendo 23 procedimentos) e 18 (14,3\%) para outros procedimentos diversos (uma fasciotomia de coxa e uma transferência tendinosa, e os outros 17 apenas para retiradas de projéteis e/ou debridamento). Um paciente, além de tratar a fratura de ossos da mão com fixador interno, foi submetido a tenorrafia e a neurorrafia na mão.

Os 23 tratamentos cirúrgicos de fraturas foram: 9 fixações internas (39,1\%); 5 fixações externas (21,8\%); 5 fixações externas seguidas de fixação interna (21,8\%); 2 artrodeses da coluna (8,7\%); 1 laminectomia descompressiva de L5-S1 (4,3\%); 1 fixação externa em quadril e fixação interna em fêmur para tratamento de fratura subtrocantérica (4,3\%).

Quanto à evolução, complicações e sequelas durante o $1^{\circ}$ atendimento hospitalar: houve 7 sequelas, sendo 1 osteonecrose de cabeça de fêmur e 6 lesões medulares ( 3 paraplegias; 1 tetraplegia; 1 hemiplegia e paraparesia de membro inferior direito; e 1 perda de força e paraparesia de membro inferior esquerdo por avulsão de raiz). Outras complicações foram: oito lesões de nervos periféricos, sendo cinco motoras e três motoras e sensitivas; quatro tiveram infecções, sendo uma osteomielite aguda; uma teve síndrome compartimental com lesão do nervo femoral. Houve um paciente que retornou por granuloma de corpo estranho no cotovelo direito e necessitou remoção da bala.

Ocorreram lesões associadas em 52 pacientes (41,3\%), em um total de 84 lesões associadas, sendo: 27 abdominais (32,1\%); 25 torácicas $(29,8 \%) ; 13$ vasculares $(15,5 \%) ; 12$ faciais (14,3\%); e 7 encefálicas (8,3\%). Dos 52 pacientes com lesões associadas, 38 foram submetidos a procedimentos de outras especialidades médicas (30,2\% dos 126 pacientes). Seis pacientes evoluíram a óbito. Nos 58 atendimentos com fraturas, 31 tiveram lesões associadas (53,5\%).

Dos 126 pacientes, $70(55,6 \%)$ foram internados, sendo que $21(16,7 \%)$ necessitaram de internação na unidade de tratamento intensivo (UTI). Os outros $56(44,4 \%)$ apenas deram entrada ao PS e receberam alta. $O$ tempo de internamento dos 70 pacientes que foram internados teve uma média de 9,6 dias, variando entre 1 e 84 dias. O tempo de internação na UTI dos 21 pacientes que necessitaram de tratamento intensivo teve uma média de 14,7 dias, variando entre 1 e 82 dias.

Um total de 120 pacientes sobreviveu (95,2\%), e 6 (4,8\%) evoluíram a óbito.

\section{Discussão}

Apesar do conhecimento geral da realidade violenta que enfrentamos no nosso país, e de que as crianças e adolescentes compõem um grupo vulnerável, existem poucos dados abordando as implicações médicas desta situação. $O$ presente estudo é, pelo nosso conhecimento, o primeiro estudo nacional que avalia lesões por armas de fogo especificamente em crianças e adolescentes (menores de 18 anos). Em um estudo realizado no Children's Hospital of Alabama, Birmingham, estado do Alabama, EUA, foram registrados 194 casos de procura por FAF em menores de 19 anos de idade em um período total de 11 anos (de abril de 1999 a março de 2010). ${ }^{12}$ Nosso estudo, com levantamento de 3 anos e menor faixa etária (18 anos incompletos), encontrou 169 vitimas (considerando 
as não musculoesqueléticas), um resultado que praticamente se iguala ao do estudo norte-americano. Salientamos que os dois hospitais têm porte semelhante e o fato de no estado do Alabama o porte de arma ser legalizado. Outro estudo, realizado no Carolinas Medical Center, em Charlotte, no estado da Carolina do Norte, nos Estados Unidos, com duração de 8 anos, mais do que o dobro do período do nosso estudo, incluindo semelhantemente apenas pacientes ortopédicos e menores de 18 anos, houve um total de 46 pacientes lesionados, ou seja, 2,75 vezes menos do que no nosso estudo. ${ }^{13}$

Vale ressaltar que vem havendo um aumento desse tipo de ocorrência no Brasil, segundo o Mapa da Violência de 2016 . $^{10}$ No conjunto da população, o número de homicídios por armas de fogo (HAF) teve um crescimento de 592,8\% entre 1980 e 2014. Contudo, na faixa jovem, este crescimento foi bem maior: $700 \%$. Esta faixa, por estar entre 15 e 29 anos de idade, inclui adolescentes, os quais são objetos do presente estudo. É justamente na adolescência que o índice aumenta exponencialmente. ${ }^{10}$ Já nos registros do DATASUS, houve 299 óbitos na cidade de Curitiba no período de 2014 a 2016. Este número elevado pode ser decorrente da faixa etária mais ampla abrangida pelo DATASUS (até 19 anos de idade) - que é justamente o pico das ocorrências, o que dificulta sua comparação com nossos dados. ${ }^{4}$ Nosso estudo apresentou a maior porcentagem também nessa parcela, embora ela seja de até 18 anos incompletos. Contudo, o início do pico se deu aos 14 anos de idade, sugerindo que as ocorrências já estejam atingindo idades mais precoces.

Os artigos internacionais, principalmente norte-americanos, enfatizam a causa das lesões (se violentas ou não intencionais). Isto se dá, principalmente, pela diferença dos estados em relação às leis de armas. Em 2014, Safavi et al ${ }^{14}$ publicaram um estudo no qual a média de lesões em menores de 18 anos de idade vivendo em um estado sem restrições para o porte de arma era 3,75 vezes maior do que em um estado com leis restritas para o porte. No nosso estudo, não pudemos obter os dados sobre motivos da lesão, embora fosse um interesse inicial. Nos estudos norte-americanos, a maioria das lesões é não intencional. ${ }^{13}$ Srinivasan et al ${ }^{15}$ publicaram em seu estudo que $62 \%$ das lesões nos EUA ocorrem não intencionalmente (foram analisadas 198.969 procuras por FAF em menores de 19 anos nos EUA, de 2001 a 2010).

Quanto à participação do ortopedista, nosso estudo mostrou que a cada quatro crianças ou adolescentes com queixa de FAF na emergência, aproximadamente três tiveram lesões musculoesqueléticas (critérios de inclusão) e necessitaram de ao menos um primeiro atendimento ortopédico. Este dado enfatiza a necessidade do ortopedista ter um conhecimento do perfil das lesões, das habilidades necessárias para o tratamento, assim como da estrutura e dos equipamentos para prover assistência adequada.

Como em outros estudos, o gênero masculino mostrou-se mais susceptível, com a proporção de $85 \%$. Em 2014, o VIVA Inquérito relatou que $72 \%$ de vítimas de violência, em todas as idades, são homens, e que isso se repete para crianças e adolescentes. $^{1}$

Em relação à idade, a média deste estudo é de 15 anos e 5 meses, variando de 2 a 17 anos, enquanto que para Perkins et al $^{13}$ os extremos também são de 2 a 17 anos de idade, mas a média é de 12,7 anos. Para Naranje et al, ${ }^{16}$ a média também $\sim$ foi $\sim 12$ anos. Aproximadamente 90\% dos pacientes do nosso estudo eram maiores de 13 anos, enquanto esta faixa é composta por $72 \%$ no estudo de Perkins et al. ${ }^{13}$ Esta divergência se dá provavelmente pelas diferenças nas proporções já comentadas entre lesões intencionais e não intencionais entre os dois países.

Metade dos nossos pacientes teve ao menos uma bala alojada. $\mathrm{O}$ procedimento de retirada de bala foi realizado em aproximadamente um terço dos pacientes. No estudo de Mazotas et al, ${ }^{17} 22 \%$ dos 107 pacientes pediátricos tiveram complicações secundárias à localização do projétil, mas os autores somente recomendam a sua retirada quando ele está localizado em articulações. Segundo Ootani et al, ${ }^{6}$ na presença de projétil na articulação, deve ser feita a remoção, pois além do projétil agir como corpo livre intra-articular, ele provoca transtornos de ordem eletrolítica. Em nosso estudo, um paciente retornou por granuloma de corpo estranho e necessitou remoção da bala, que estava localizada em uma junta (cotovelo).

Na literatura, quase na metade das vezes em que os membros são atingidos, ocorrem fraturas. ${ }^{10}$ Em nosso estudo, o índice foi de $46 \%$. Nove (15\%) apresentaram múltiplas fraturas, índice menor que o encontrado por Naranje et al $(18,4 \%) .{ }^{16}$ Nosso artigo mostra que os membros inferiores são os mais atingidos (56\%), o que corrobora o encontrado em

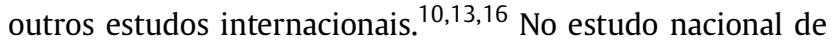
Ootani et al, 6 envolvendo lesões ósseas por armas de fogo em idades entre 5 e 54 anos, os membros inferiores também foram os mais atingidos, mas a proporção deles foi maior ( $\sim 65 \%)$.

Além disso, a coxa foi o principal compartimento atingido, e o fêmur e tíbia tiveram incidências semelhantes de lesões (15\% e 14\%, 11 fraturas no fêmur e 10 na tíbia), e isso também ocorreu nos estudos de Perkins et al ${ }^{13}$ e de Naranje et al. ${ }^{16}$ No estudo brasileiro citado, o fêmur foi muito mais atingido do que a tíbia; o que pode sugerir uma diferença na epidemiologia entre as faixas etárias. Já a mão, assim como no presente estudo, foi o local do membro superior mais atingido. ${ }^{6}$

Outro importante segmento avaliado, pelo seu potencial de sequelas graves, foi a coluna. Quinze pacientes apresentaram 22 fraturas vertebrais ( 8 torácicas, 7 lombares e 5 cervicais). Em um estudo de 2011, também na cidade de Curitiba, que avalia traumas raquimedulares por FAF em todas as idades, o nível mais acometido também foi o torácico (42,6\%); mas o nível cervical teve mais que o dobro de traumas do que o lombar (37,1\% e $16,6 \%$, respectivamente), ${ }^{8}$ semelhantemente ao estudo de Barros Filho et $\mathrm{al}^{9}$ (também de FAF em coluna em todas as idades), e diferentemente do nosso. Já na literatura estrangeira, em Naranje et al, ${ }^{16}$ ocorreu apenas uma fratura de coluna (que foi na vértebra $\mathrm{C} 5$ ). Seis dos nossos pacientes apresentaram lesões medulares definitivas. Carter et $\mathrm{al}^{11}$ relataram que a maioria das fraturas espinhais não necessita de tratamento cirúrgico, e que a realização de cirurgia não é associada a melhor prognóstico neurológico. Na literatura nacional, Barros Filho et $\mathrm{al}^{5}$ afirmaram que o tratamento cirúrgico de rotina no trauma de coluna não parece adequado. Dos nossos 15 pacientes com lesões, apenas 3 foram submetidos a cirurgia. 
Em nosso estudo, a maioria dos pacientes foi tratada de forma conservadora (63\%), divergindo de Perkins et al, ${ }^{13}$ no qual pouco mais da metade (52\%) foi tratada de forma cirúrgica. Dos pacientes tratados cirurgicamente, aproximadamente metade foi para estabilização de fraturas $(n=19)$ e os demais para debridamento, retirada de projéteis e outros procedimentos. De modo semelhante a outros estudos, ${ }^{13,16}$ cerca de dois terços das fraturas necessitaram fixação interna, e $26 \%$ necessitaram fixação externa.

Outras lesões com importante potencial de sequelas foram observadas. Lesões de nervo periférico $(6,3 \%)$ foram menos comuns do que no estudo de Perkins et al, ${ }^{13}$ (com 13\%). Apenas uma lesão em nosso estudo evoluiu para síndrome compartimental. Um ponto comentado por outros autores é que apesar do FAF ser uma fratura exposta, a osteomielite não é comum após tratamento de $\mathrm{FAF}^{16}$ e nós observamos apenas um caso em nossa série. Quatro pacientes evoluíram com infecção (3\%), índice inferior ao de Perkins et al $(13 \%)^{13} \mathrm{Um}$ fator balístico pode ser relevante: Naranje et $\mathrm{al}^{16}$ encontraram $100 \%$ de infecção em ferimentos causados por espingarda, e menos de $10 \%$ nos causados por pistola. Além da coluna, vale atentarse às articulações em que os danos podem ser bastante variáveis. Um dos nossos pacientes, atingido na articulação coxo-femoral, evoluiu com necrose avascular da epífise.

O tratamento de vítima de arma de fogo deve ser multidisciplinar, com a participação do cirurgião geral, vascular, neurocirurgião e ortopedista. ${ }^{7}$ Um total de 52 pacientes teve lesões associadas ( $41 \%)$, um índice parecido com o de Naranje et al. ${ }^{16} \mathrm{~A}$ maioria das lesões correspondeu a lesões abdominais e torácicas, o que difere de um extenso estudo retrospectivo norte-americano de FAF em menores de idade, no qual as lesões encefálicas foram a segunda mais frequente (atrás apenas das musculoesqueléticas). ${ }^{12}$ Uma possibilidade para essa divergência é que o estudo norte-americano afirma que lesões encefálicas são proporcionalmente mais frequentes em menores de 9 anos de idade, e no nosso estudo apenas $10 \%$ dos pacientes estavam nesta faixa etária. Outro fato é que a taxa de óbitos deste estudo foi de $9,3 \%,{ }^{12}$ superior à nossa, sugerindo uma epidemiologia diferente.

Aproximadamente $55 \%$ dos nossos pacientes foram internados, e o tempo médio de internação foi superior aos dos estudos de Perkins et $\mathrm{al}^{13}$ e de Naranje et $\mathrm{al}^{16}$ (9,6 versus 6,8 versus 5,8 dias, respectivamente). A evolução para óbito não é frequente, ainda mais se tratando de lesões exclusivamente musculoesqueléticas, pois todos os seis pacientes que faleceram apresentavam lesões associadas. 0 presente estudo apresentou uma incidência de $\sim 4,5 \%$ de óbitos, o que é um pouco superior às de outros estudos, ${ }^{15,18}$ mas, como já referido, é inferior ao estudo de Senger et al (9,3\%). ${ }^{12}$

Nosso estudo apresenta limitações, como a ausência de informações que poderiam ser importantes: dados gerais como motivo, raça e dados de balística; assuntos que são tratados em artigos de referência. ${ }^{10,13,16,18}$ Devido a um índice de perda de seguimento importante, optamos por focar somente no atendimento emergencial. Um acompanhamento mais longo, além da avaliação do resultado final, pode demonstrar o aparecimento de outras potenciais complicações. No entanto, pela ausência de outros estudos nacionais, consideramos importante iniciar um estudo com dados mais básicos.

\section{Conclusão}

O atendimento ortopédico está presente na maioria das crianças e adolescentes que são encaminhados ao pronto socorro por FAFs. Os adolescentes e o sexo masculino são o grupo de maior risco, e as lesões ocorrem principalmente em membros inferiores. Mais da metade dos pacientes precisou ser internada.

O tratamento conservador foi possível na maioria das fraturas, e a mortalidade dos pacientes é relativamente baixa. Apesar de menos da metade dos pacientes terem apresentado fraturas, muitos apresentaram lesões complexas com potencial de graves sequelas.

\section{Conflitos de Interesses}

Os autores declaram não haver conflitos de interesses.

\section{Agradecimentos}

Ivone da Silva Costa e Maria Barbosa, funcionárias do Hospital do Trabalhador, pelo auxílio para levantamento de dados de atendimento.

Ao acadêmico Danilo Celeste pelo auxílio no acesso aos dados digitais.

Ao dr. Weverley Valenza, chefe do setor de Ortopedia do Hospital do Trabalhador, pelo apoio para a realização do presente estudo.

\section{Referências}

1 Souto RMCV, Barufaldi LA, Nico LS, Freitas MG. Perfil epidemiológico do atendimento por violência nos serviços públicos de urgência e emergência em capitais brasileiras, Viva 2014. Cien Saude Colet 2017;22(09):2811-2823

2 Fraga AM, Bustorff-Silva JM, Fernandez TM, et al. Children and adolescents deaths from trauma-related causes in a Brazilian City. World J Emerg Surg 2013;8(01):52

3 Malta DC, Souza ER, Silva MM, et al. Vivência de violência entre escolares brasileiros: resultados da Pesquisa Nacional de Saúde do Escolar (PeNSE). Cien Saude Colet 2010;15(02, Suppl 2):3053-3063

4 Brasil. SIM/DATASUS/MS. O Sistema de Informações sobre Mortalidade. S/l, 1995. Disponível em: http://www2.datasus.gov.br/DATA SUS/index.php? area $=0205 \& i d=6937 \& V O b j=h t t p: / /$ tabnet.datasus. gov.br/cgi/deftohtm.exe?sim/cnv/obt10

5 Barros Filho TE, Mendonça Neto AB, Oliveira RP, Taricco MA. Traumatismo na coluna vertebral por projéteis de arma de fogo. Rev Bras Ortop 1989;24(06):190-192

6 Ootani SO, Silva RJ, Santos MA, Yamamura Y. Lesões ósseas provocadas por projéteis de arma de fogo. Rev Bras Ortop 1984; 19(02):66-72

7 Domit K. Traumatismo músculo-esquelético por projétil de arma de fogo. Rev Bras Ortop 1997;32(10):827-831

8 Araújo JF, Heinrich CB, Cunha ML, et al. Traumatismo raquimedular por ferimento de projétil de arma de fogo: avaliação epidemiológica. Coluna/Columna 2011;10(04):290-292

9 Barros Filho TE, Oliveira RP, Barros EK, Von Uhlendorff EF, Iutaka AS. Ferimentos por arma de fogo na coluna vertebral: estudo epidemiológico. Coluna/Columna 2002;1(02):83-87

10 Waiselfisz JJ. Mapa da Violência 2016: Homicídios por Armas de Fogo. Rio de Janeiro: FLACSO/CEBELA; 2016 
11 Carter CW, Sharkey MS, Fishman F. Firearm-related Musculoskeletal Injuries in Children and Adolescents. J Am Acad Orthop Surg 2017;25(03):169-178

12 Senger C, Keijzer R, Smith G, Muensterer OJ. Pediatric firearm injuries: a 10-year single-center experience of 194 patients. J Pediatr Surg 2011;46(05):927-932

13 Perkins C, Scannell B, Brighton B, Seymour R, Vanderhave K. Orthopaedic firearm injuries in children and adolescents: An eight-year experience at a major urban trauma center. Injury 2016;47(01):173-177

14 Safavi A, Rhee P, Pandit V, et al. Children are safer in states with strict firearm laws: a National Inpatient Sample study. JTrauma Acute Care Surg 2014;76(01):146-150, discussion 150-151
15 Srinivasan S, Mannix R, Lee LK. Epidemiology of paediatric firearm injuries in the USA, 2001-2010. Arch Dis Child 2014;99 (04):331-335

16 Naranje SM, Gilbert SR, Stewart MG, et al. Gunshot-associated Fractures in Children and Adolescents Treated at Two Level 1 Pediatric Trauma Centers. J Pediatr Orthop 2016;36(01):1-5

17 Mazotas IG, Hamilton NA, McCubbins MA, Keller MS. The longterm outcome of retained foreign bodies in pediatric gunshot wounds. J Trauma Nurs 2012;19(04):240-245

18 Veenstra M, Patel V, Donoghue L, Langenburg S. Trends in pediatric firearm-related injuries over the past 10 years at an urban pediatric hospital. JPediatr Surg 2015;50(07):11841187 